\title{
Endoscopic suture repair of full-thickness esophagotomy during per-oral esophageal myotomy for achalasia
}

\author{
Ashwin A. Kurian • Neil H. Bhayani • \\ Kevin Reavis · Christy Dunst · Lee Swanström
}

Received: 22 August 2012/Accepted: 26 April 2013/Published online: 25 May 2013

(C) Springer Science+Business Media New York 2013

\begin{abstract}
Background Per-oral endoscopic myotomy (POEM) requires advanced flexible endoscopic skills, especially in the management of complications.

Methods We present a full-thickness esophagotomy while performing POEM and repair using an endoscopic suturing device.

Standard operative technique An anterior esophageal $2 \mathrm{~cm}$ mucosectomy is created $7-10 \mathrm{~cm}$ proximal to the gastroesophageal junction after a submucosal wheal is raised. A submucosal tunnel is created and extended to $2 \mathrm{~cm}$ on the gastric cardia. A selective circular myotomy is performed. The mucosectomy is closed using endoscopic clips.

Case presentation An inadvertent full-thickness esophagotomy was created while performing the mucosotomy on an inadequate submucosal wheal. We were able to resume the POEM technique at the initial esophagotomy site. There was a discussion to convert to laparoscopy. However, as we succeeded in creating the tunnel, we continued
\end{abstract}

Presented at the SAGES 2012 Annual Meeting, March 7-March 10, 2012, San Diego, CA.

Electronic supplementary material The online version of this article (doi:10.1007/s00464-013-3002-8) contains supplementary material, which is available to authorized users.

A. A. Kurian $(\varangle) \cdot$ N. H. Bhayani

Department of General Surgery, Providence Portland Cancer

Center, Portland, OR, USA

e-mail: ashwinkurian@hotmail.com

K. Reavis · C. Dunst $\cdot$ L. Swanström

Gastrointestinal and Minimally Invasive Surgery Division,

The Oregon Clinic, 4805 NE Glisan Street, \#6N60, Portland,

Oregon 97213, USA

e-mail: 1swanstrom@aol.com with the POEM technique. After the selective myotomy was completed, we used an endoluminal suturing device (Overstitch, Apollo Endosurgery, Austin TX) to close the full-thickness esophagotomy in two layers (muscular, mucosal). A covered stent was not an option because the esophagus was dilated, which precluded adequate apposition. The patient had an uneventful postoperative course. At 9-month follow-up, had excellent palliation of dysphagia without reflux.

Conclusions This case demonstrates the importance of identifying extramucosal intrathoracic anatomy, thus emphasizing the need for an experienced surgeon to perform these procedures, or at a minimum to be highly involved. Raising an adequate wheal is crucial before mucosectomy. Inadequacy of the wheal may reflect local esophageal fibrosis. If this fails at multiple locations in the esophagus, it may be prudent to convert to laparoscopy. This case also demonstrates the need for advanced flexible endoscopic therapeutic tools and a multidisciplinary approach to manage potential complications.

Keywords Abdominal - Achalasia - Digestive . Endoscopy $\cdot$ Esophageal POEM

Disclosure Drs. Kurian, Bhayani, Reavis, Dunst, and Swanström have no conflicts of interest or financial ties to disclose. 